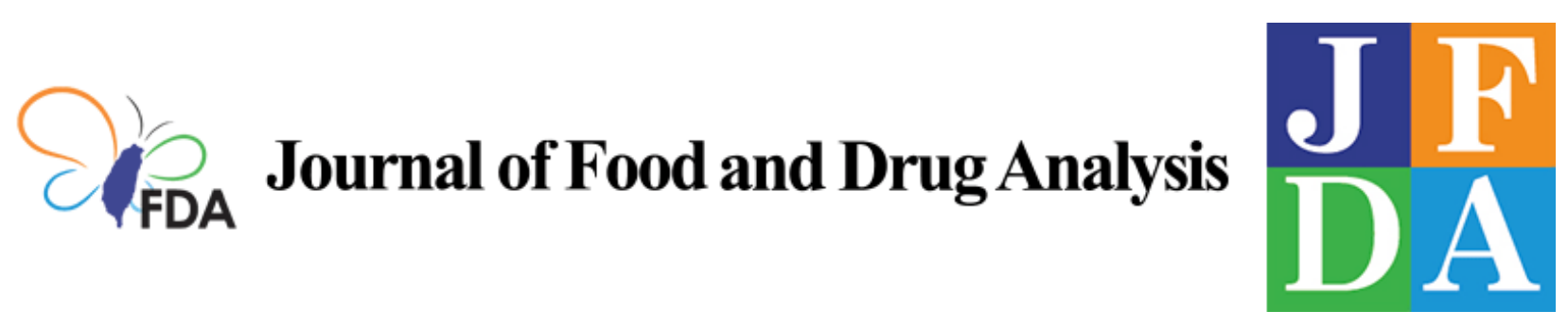

Volume 28 | Issue 2

Article 12

2020

\title{
Preparation, evaluation and metabolites study in rats of novel Isoginkgetin-loaded TPGS/soluplus mixed nanomicelles
}

Follow this and additional works at: https://www.jfda-online.com/journal

Part of the Food Science Commons, Medicinal Chemistry and Pharmaceutics Commons, Pharmacology Commons, and the Toxicology Commons

(c) (i) (9)

This work is licensed under a Creative Commons Attribution-Noncommercial-No Derivative Works 4.0 License.

\section{Recommended Citation}

Feng, Xue; Chen, Yu-Ting; Li, Lu-Ya; Sun, Yu-Peng; Wang, Hai-Rong; and Zhang, Lan-Tong (2020)

"Preparation, evaluation and metabolites study in rats of novel Isoginkgetin-loaded TPGS/soluplus mixed nanomicelles," Journal of Food and Drug Analysis: Vol. 28 : Iss. 2 , Article 12.

Available at: https://doi.org/10.38212/2224-6614.1065

This Original Article is brought to you for free and open access by Journal of Food and Drug Analysis. It has been accepted for inclusion in Journal of Food and Drug Analysis by an authorized editor of Journal of Food and Drug Analysis. 


\title{
Preparation, evaluation and metabolites study in rats of novel Isoginkgetin-loaded TPGS/soluplus mixed nanomicelles
}

\author{
Xue Feng, Yu-Ting Chen, Lu-Ya Li, Yu-Peng Sun, Hai-Rong Wang, Lan-Tong Zhang*
}

Department of Pharmaceutical Analysis, School of Pharmacy, Hebei Medical University, No.361, Zhongshan Road, Shijiazhuang 050017, PR China

\begin{abstract}
At present, cancer is one of the most lethal diseases in the world, and researchers are committed to developing effective anticancer drugs. Isoginkgetin (IGG) is a kind of biflavone with the potential to treat cancer due to the features of altering the cell cycle and inhibiting tumor cell infiltration. However, its solubility, absorbability and bioavailability are poor, so in this study, IGG was prepared into mixed nanomicelles and evaluated in vitro and in vivo. After condition optimization, IGG-loaded TPGS/soluplus mixed nanomicelles with particle size of $62.34 \pm 1.10 \mathrm{~nm}$, entrapment efficiency of $96.92 \pm 0.66 \%$ and drug loading of $2.42 \pm 0.02 \%$ were successfully prepared. The physicochemical properties of the nanomicelles were stable within 60 days, and the cytotoxicity of the nanomicelles was significantly higher than that of IGG. The metabolism results showed that 32 kinds of metabolites of IGG and 21 kinds of IGG-loaded nanomicelles were detected. The metabolites of IGG can only be detected in feces of rats, while the metabolites of IGG-loaded nanomicelles can be detected in plasma, bile, urine and feces. All these indicated that after prepared into nanomicelles, the stability, solubility, cytotoxicity and bioavailability of IGG were increased significantly, which provided a new choice for the development of new drugs.
\end{abstract}

Keywords: Isoginkgetin, Nanomicelle, Cytotoxicity, Cellular uptake, Metabolite

\section{Introduction}

A $t$ present, cancer is one of the most lethal diseases in the world. The main way to treat cancer is surgical treatment, adjuvant chemotherapy, radiotherapy, and traditional Chinese medicine treatment. However, due to the large side effects of chemotherapy and radiotherapy, the patient's postoperative quality of life is poor, and the cure rate of cancer is not high [1]. Therefore, the researchers all over the world are working to find medicines that can quickly and effectively treat cancer, and strive to reduce the side effects of medicines. Traditional Chinese medicine with the advantages of low side effects and fast curative effect, it has attracted much attention in recent years. Paclitaxel [2], ginsenoside Rg3 [3], and resveratrol [4] have been clinically proven to have good anti-cancer effects.

Biflavones, as a special natural active component of flavonoids, its content in nature is very small, mainly in gymnosperms such as Ginkgo biloba, Selaginella, Platycladus orientalis, Selaginella chinensis and Taxus canadensis. Compared with monoflavones, biflavones exhibit different pharmacological activities. In some aspects, their activities are higher than monoflavones [5]. In view of this, biflavones have good development prospects. Isoginkgetin (IGG) is a kind of biflavone extracted from Ginkgo biloba leaves. It has antimicrobial [6], antioxidant [5] and free radical scavenging activities. It can change cell cycle [7], inhibit the infection of tumor cells [8], and has the potential to treat cancer

Received 8 January 2020; accepted 21 February 2020.

Available online 27 June 2020

* Corresponding author at: Department of Pharmaceutical Analysis, School of Pharmacy, Hebei Medical University, No.361, Zhongshan Road, Shijiazhuang 050017, China. Fax: +86-0311-86266419.

E-mail address: zhanglantong@263.net (L.-T. Zhang). 
[9]. In addition to its ability to inhibit pre-mRNA splicing [10], IGG can also disrupt protein homeostasis, cause excess protein in cells which increasing the burden on lysosomal/autophagic mechanisms, and cause cancer cell death [11]. Moreover, IGG slowed the progression of the cell cycle and reduced the number of metaphase cells during multiple phases of the cell cycle (G1, S, and G2). S-phase blockade was the main effect detected after IGG treatment [7]. Isoginkgoside is less toxic to rat skeletal muscle myoblasts cultured in vitro. Its mechanism of action is to inhibit tumor cell invasion by regulating the expression of phosphatidylinositol 3-kinase/Akt-dependent matrix metalloproteinase-9 [8]. Therefore, IGG has considerable research prospects in the treatment of cancer.

Previous studies have shown that the bioavailability of biflavones is generally low, and drugs can't be effectively used by organisms after oral administration. In order to give full play to its pharmacological role, it is necessary to improve its bioavailability. At the same time, to improve patient comfort and compliance, oral administration is a better choice, especially for long-term administration. However, many antitumor drugs have low solubility, poor stability in the severe physiological state of the gastrointestinal tract, and low permeability of the intestinal epithelium. In order to solve the above-mentioned dilemma, the nanoparticlebased drug delivery system has received special attention [12]. In recent years, micelle-based drug delivery systems have been extensively developed due to their enhanced pharmacokinetics, biodistribution, and high stability [13], and oral bioavailability has been greatly improved after micelle preparation [14]. Therefore nanomicelles were prepared and evaluated in vitro and in vivo.

Compared to free drugs, nanoparticle therapeutics has been shown to have enhanced drug accumulation in solid tumors, resulting in better anticancer activity [15]. In addition, particles with a particle size of $20-200 \mathrm{~nm}$ can avoid the filtering effect of glomeruli and tubules, and are targeted to tumors, so they can reduce systemic toxicity [16]. And the absorption of nanoparticle drugs must also overcome the mucosal diffusion barrier and epithelial absorption barrier, which requires the particle surface be close to electrically neutral and have a particle size of less than $200 \mathrm{~nm}$ [17]. Therefore, in this study, we were committed to preparing nanomicelles that meet the above requirements.

Mixed nanomicelles have many advantages over single nanomicelles. First, due to the hydrophobicity of the core [18], these substances are particularly suitable for the dissolution of water-insoluble drugs, and also can protect unstable drugs from biochemical degradation and metabolism, thus enhancing the solubility and stability of the drugs. Secondly, Polymeric micelles are kinetically stable so they dissociate slowly, even at concentrations below the critical micelle concentration (CMC), extending circulation time in blood [19]. Thirdly, mixed micelle system can reduce the inconsistency and non-specific uptake of the reticuloendothelial system, and enhance the targeting of the drug by enhancing the permeability and retention effect [20]. In addition, the size of mixed micelles range from 20 to $200 \mathrm{~nm}$, which is large enough to avoid rapid elimination of renal tubules, and also small enough to penetrate blood vessels, enhance drug targeting and reduce toxicity to non-specific organs. Besides, mixed micelles can also reduce adverse effects of drugs [21], increase drug loading and delay drug release, so they have been widely used in recent years, and have considerable research prospects [22].

In this experiment, the mixed micelles of IGG were prepared by membrane hydration method using polyvinyl caprolactam-polyvinyl acetate-polyethylene glycol graft copolymer (soluplus) and $D-\alpha$-tocopheryl polyethylene glycol 1000 succinate (TPGS) as carriers. Soluplus has good solubilization for poorly water-soluble drugs, and can reduce the $C M C$ value and increase the drug loading [22]. However, in recent years, there have been few studies on the use of soluplus to improve the solubility of drugs, so the research prospect of soluplus is very broad. TPGS has been widely used in the preparation of pharmaceutical formulation products as an absorption enhancer, antioxidant, emulsifier, solubilizer, surfactant, suspending agent [23], and has been reported that it can inhibit the effect of P-gp, and promote cell apoptosis and show certain toxicity to cancer cells [22].

\section{Materials and methods}

\subsection{Chemicals and materials}

IGG (DST180423-191, purity > 98\%) was purchased from Chengdu Desite Biotech Co., Ltd. Vitamin E polyethylene glycol succinate (TPGS) was purchased from Shanghai Yuanye Bio-Technology Co., Ltd. Soluplus is purchased from BASF (Germany). HPLC - grade acetonitrile and methanol were purchased from American J.T.-Baker Chemical Company (Phillipsburg, NJ, USA). HPLC-grade formic acid was provided by Diamond Technology (Dikma Technologies Inc., Lake Forest, CA, USA). 
And analytical pure methanol was purchased from Tianjin Baishi Chemical Co., Ltd. CCK-8 was purchased from Beijing Zoman Biotechnology Co., Ltd. Heparin was purchased from Changzhou Qianhong Bio-pharma Co., Ltd. Phosphate buffer (PBS), were purchased from Sigma Chemical Co. (St. Louis, MO, USA). Dimethyl sulfoxide (DMSO) iodine $\left(\mathrm{I}_{2}\right)$, potassium iodide (KI) and sodium carboxymethyl cellulose (CMC-Na) were purchased from Tianjin Yongda Reagent Co., Ltd. Purified water was purchased from Hangzhou Wahaha Group Co., Ltd.

\subsection{Methods}

\subsubsection{Preparation of mixed micelle}

The preparation of IGG-loaded TPGS/soluplus mixed micelles was carried out by film hydration method [24]. In short, IGG (2 mg), soluplus (60 mg), TPGS $(20 \mathrm{mg})$ was precisely weighed and dissolved in $20 \mathrm{~mL}$ methanol in a round bottom flask by stirring at room temprature. Then, the solvent was vacuum evaporation by EYELA N1100 rotary evaporator (Tokyo Rikakikai Co., Ltd., Japan) at $37^{\circ} \mathrm{C}$ for $1 \mathrm{~h}$ to form a layer of film. Next, $8 \mathrm{~mL}$ water was added and hydration in ice water by EQ5200E ultrasonic cleaner (Kun Shan Ultrasonic Instruments Co., Ltd) for $1 \mathrm{~h}$ to obtain a clear micelle solution. Subsequently, the solution was ultrasonic crashed for 5 min by Ultrasonic crushing instrument (Wuxi Voshin instruments Manufacturing Co., Ltd.). Finally, the micelle solution was filtered by $0.22 \mu \mathrm{m}$ filter membrane to remove unloaded drug. The blank micelles were prepared by the same method.

\subsubsection{Determination of critical micelle concentration (CMC)}

CMC is a very important index to evaluate formation ability of micelles. Lower CMC indicated higher formation ability and higher stability of micelles. In this experiment, the CMC of IGGloaded TPGS/soluplus mixed nanomicelles was measured using the iodine hydrophobic probe method and measured by T9S dual-beam ultraviolet-visible spectrophotometer (Beijing Persee General Instrument Co., Ltd, China). The soluble iodine, a small hydrophobic molecule, preferred located in the hydrophobic microenvironment of the micelles, resulting in the conversion of $\mathrm{I}^{-}$to $\mathrm{I}_{2}$ in the solution. And the ultraviolet absorption changed can be measured with an ultraviolet spectrophotometer. Firstly, $0.5 \mathrm{~g}$ of $\mathrm{I}_{2}$ and $1 \mathrm{~g}$ of $\mathrm{KI}$ were dissolved in $50 \mathrm{~mL}$ of deionized water to prepare $\mathrm{I}_{2} / \mathrm{KI}$ standard solutions. Then TPGS/soluplus solutions with a concentration ranged from $0.00001 \%$ to $0.2 \%$ were prepared, and $25 \mu \mathrm{L}$ of $\mathrm{I}_{2} / \mathrm{KI}$ standard solution was added. Next, the mixtures were equilibrated at room temperature for $12 \mathrm{~h}$ in the dark. Finally, the ultraviolet absorbance value of each variant polymer concentrations were measured at $366 \mathrm{~nm}$ with a UV spectrophotometer. Plot the absorption intensity against the logarithm of the polymer mass concentration. The turning point of the curve was the CMC value of the mixed nanomicelles [25].

\subsubsection{Particle size and Zeta potential analysis}

The particle size and Zeta potential of IGG-loaded TPGS/soluplus mixed micelles were measured by dynamic light scattering technique using Nano-ZS particle size tester (Malvern UK). The results were expressed as mean size \pm standard deviation (SD). Each sample was measured for three times.

\subsubsection{Drug loading and encapsulation efficiency}

The encapsulation efficiency (EE) and drug loading (DL) were determined by high performance liquid chromatography (Ultimate3000, Thermo Fisher Scientific). The samples were separated on an Agilent ZORBAX SB-C18 reversed-phase chromatographic column $(5 \mu \mathrm{m}, 4.6 \mathrm{~mm} \times 150 \mathrm{~mm})$. Water (A, 0.1\% formic acid) and acetonitrile (B) was used as mobile phase, and the flow rate was $1 \mathrm{~mL} /$ min. Gradient elution was applied, and the elution procedures were as follows: $48-60 \%$ B $(0-10 \mathrm{~min})$, $60-48 \%$ B (10-10.1 $\mathrm{min}), 48 \%$ B (10.1-15 $\mathrm{min})$. The sample solution was injected at a volume of $20 \mu \mathrm{L}$. The detection wavelength was $330 \mathrm{~nm}$.

For the determination of encapsulation efficiency: The mixed micelles $(200 \mu \mathrm{L})$ was dissolved in $2 \mathrm{~mL}$ methanol, and then sonicated for $10 \mathrm{~min}$ to release the encapsulated drug. The solution was then centrifuged twice at $21,380 \times g$ for $10 \mathrm{~min}$. Take the supernatant sample for analysis.

For the determination of drug loading: First, the prepared IGG-loaded micelle solution was lyophilized, and $45 \mathrm{mg}$ of the lyophilized powder was dissolved in $1 \mathrm{~mL}$ water. Then $200 \mu \mathrm{L}$ of the dissolved micelle solution was added to $2 \mathrm{~mL}$ methanol, and treated with ultrasonic for about $10 \mathrm{~min}$ until the encapsulated drug was released. Finally, the solution was centrifuged twice at $21,380 \times g$ for $10 \mathrm{~min}$, and the supernatant was taken for analysis. 
The determination of the concentration of IGG in the mixed micelles was carried out by external standard method. The formula for calculating the encapsulation efficiency and drug loading was as follows:

$$
\begin{aligned}
& \mathrm{EE} \%=\mathrm{W}_{\mathrm{IGG}} / \mathrm{W}_{\mathrm{IGG}} \times 100 \% \\
& \mathrm{DL} \%=\mathrm{W}_{\mathrm{IGG}} / \mathrm{W}_{\text {micelle }} \times 100 \%
\end{aligned}
$$

$\mathrm{W}_{\mathrm{IGG}}$ : the weight of drug in micelles; $\mathrm{W}_{\mathrm{IGG}}$ : the weight of feeding drug;

$W_{\text {micelle: }}$ the total weight of feeding soluplus, TPGS and drug in micelles.

\subsubsection{Optimization of preparation conditions}

Because the ratio of drugs to excipients has a great influence on the size of micelles, encapsulation efficiency and drug loading, in this experiment the ratio of excipients to IGG was optimized with particle size, dispersion coefficient (PDI), Zeta potential and entrapment efficiency as indicators. The results are shown in Table 1.

\subsubsection{Micelle stability}

The micelle solution of IGG prepared by the method of "2.2.1." was stored at $4{ }^{\circ} \mathrm{C}$ for 60 days. The particle size, dispersion coefficient (PDI), Zeta potential and encapsulation efficiency of the IGGloaded micelles were measured at $0,15,30$ and 60 days, respectively. The clarity of the micelle solution was observed.

\subsubsection{Cell culture}

Human lung cancer line A549 cells (obtained from Shanghai cell bank of Chinese Academy of Sciences) were used in the cytotoxicity and cellular uptake experiments of IGG-loaded micelles. A549 cells were cultured in F-12 medium containing 1\% penicillin-streptomycin and $10 \%$ fetal bovine serum and placed in HF240 cell culture box (Shanghai Lishen Scientific Instrument Co., Ltd) with a constant temperature of $37^{\circ} \mathrm{C}$ and a $\mathrm{CO}_{2}$ concentration of $5 \%$.

\subsubsection{In vitro cytotoxicity}

In vitro cytoxicity of IGG-loaded TPGS/soluplus mixed micelles was performed by CCK-8 assay. Briefly, A549 cells were trained in 96 well plates at the concentration of 5000 cells/well. After $24 \mathrm{~h}$ of incubation, the cells were co-cultured with drugs for another $24 \mathrm{~h}$. Then, $10 \mu \mathrm{L}$ CCK-8 was added and cultured for $2 \mathrm{~h}$ before tested at $450 \mathrm{~nm}$ by SpectraMax Plus384 Molecular Devices (Molecular Devices, USA). Each concentration was repeated in triplicate. GraphPad Prism 5 was used to calculate the $\mathrm{IC}_{50}$ value of IGG and the IGG-loaded TPGS/ soluplus mixed micelles.

\subsubsection{In vitro cellular uptake}

The content of IGG in A549 cells was determined by HPLC method. A549 cells in good growth condition were inoculated into 6-well plates at the concentration of $5 \times 10^{5}$ cells/well. They were cultured overnight at $37{ }^{\circ} \mathrm{C}$ for $24 \mathrm{~h}$. Then the old medium was sucked out and washed three times with PBS. The prepared medium containing drugs $(30 \mu \mathrm{g} / \mathrm{mL})$ was added. The cells were cultured at $37{ }^{\circ} \mathrm{C}$ for $1,2,4$ and $6 \mathrm{~h}$. The control group was treated with no drugs. Three replicates were made for each sample. After incubation, the cells were washed with PBS three times, and $0.25 \mathrm{~mL}$ trypsin was added to each well. After digestion, the cells were collected in EP tube. After centrifugation at 157 $\times g$ for $5 \mathrm{~min}$, the supernatant was removed, and the cells were suspended with $200 \mu \mathrm{L}$ PBS. The cells were broken with ultrasonic for $5 \mathrm{~min}$ and centrifuged at $21,380 \times g$ for $10 \mathrm{~min}$. IGG content in the supernatant was measured by HPLC method and the protein content was also determined using BCA protein assay kit according to the manufacturer's protocol [22].

Cellular uptake of IGG = Intracellular IGG concentration $(\mu \mathrm{g} / \mathrm{mL}) /$ intracellular protein concentration $(\mathrm{mg} / \mathrm{mL})$

In addition, in order to observe the cellular uptake of IGG and IGG-loaded mixed nanomicelles more directly, the drug uptake process was observed by FV1200MPE laser confocal microscope (Olympus, Japan). A549 cells in good growth condition were inoculated in a 4-compartment culture dish with a density of $1.5 \times 10^{5} /$ well, cultured overnight at $37{ }^{\circ} \mathrm{C}$. After $24 \mathrm{~h}$, the old medium was removed, washed with PBS for three times, and the prepared medium $(30 \mu \mathrm{g} / \mathrm{mL})$ was added. The cells cultured continuously at $37^{\circ} \mathrm{C}$ for $6 \mathrm{~h}$, and the fluorescence

Table 1. Characteristics of IGG-loaded TPGS/Soluplus mixed nanomicelles prepared by different ratios of soluplus and TPGS.

\begin{tabular}{lllll}
\hline IGG:TPGS:soluplus & Size $(\mathrm{nm})$ & PDI & Zeta $(\mathrm{mV})$ & EE $(\%)$ \\
\hline $1: 5: 15$ & $73.05 \pm 2.23$ & $0.112 \pm 0.003$ & $-1.66 \pm 0.06$ & $57.99 \pm 0.12$ \\
$1: 10: 30$ & $62.34 \pm 1.10$ & $0.054 \pm 0.003$ & $-0.35 \pm 0.18$ & $96.92 \pm 0.66$ \\
$1: 20: 20$ & $53.67 \pm 0.34$ & $0.129 \pm 0.024$ & $0.28 \pm 0.20$ & $71.72 \pm 0.37$ \\
\hline
\end{tabular}


intensity and morphology were observed under the laser confocal microscope at $1 \mathrm{~h}, 2 \mathrm{~h}, 4 \mathrm{~h}$ and $6 \mathrm{~h}$.

\subsubsection{Metabolite analysis of IGG and IGG-loaded TPGS/soluplus mixed micelles}

2.2.10.1. Instrumentation and conditions. A Shimazu's ultra-high performance liquid chromatography system (Shimazhu 20A, Shimadzu Corporation, Kyoto, Japan) equipped with a triple TOFTM $5600^{+}$ MS/MS system (AB SCIEX, CA, USA) was applied to the metabolites determination of IGG. Samples were separated on Poroshell 120 EC-C18 $(2.1 \times 100 \mathrm{~mm}, 2.7 \mu \mathrm{m})$ with a Security Guard ${ }^{\circledR}$ UHPLC $\mathrm{C}_{18}$ pre-column (Poroshell). The mobile phase was composed of water (A, $0.1 \%$ formic acid) and acetonitrile (B), and the elution programs were as follows: $20-69 \%$ B $(0-15 \mathrm{~min}), 69-95 \% \quad \mathrm{~B}$ (15-20 $\mathrm{min}), 95 \% \mathrm{~B}(20-25 \mathrm{~min})$. The flow rate and injection volume were set at $0.3 \mathrm{~mL} / \mathrm{min}$ and $2 \mu \mathrm{L}$, respectively. The column temperature was maintained at $40{ }^{\circ} \mathrm{C}$, and the automatic injector temperature was kept at $4{ }^{\circ} \mathrm{C}$.

Mass spectrometric detection was carried out by a Triple TOFTM 5600 system with Duo-Spray ${ }^{\mathrm{TM}}$ ion sources, which was operated in the negative electrospray ionization mode. The optimized conditions of mass spectrometry as follows: ion source voltage $-4.5 \mathrm{kV}$; ion source temperature, $550{ }^{\circ} \mathrm{C}$; declustering potential (DP), $-60 \mathrm{~V}$; collision energy (CE), $-30 \mathrm{eV}+15 \mathrm{eV}$. Atomization gas (Gas 1, N2), heat gas (Gas 2, N2) and curtain gas pressure was 55 psi, 55 psi and 35 psi, respectively. The parent ion and the daughter ion scanning range were 100-1000 $\mathrm{Da}$ and 50-800 Da, respectively. Data were collected in IDA mode, and an automated calibration delivery system (CDS) was used to calibrate the MS and MS/MS automatically. The data was collected using the Analyst TF 1.6.1 software (AB SCIEX, CA, USA) for $25 \mathrm{~min}$.

\subsubsection{Animals and drug administration.} Sprague-Dawley (SD) male rats (Certificate NO. $1811164,200 \pm 20 \mathrm{~g}$ ) were provided by the experimental animal center of Hebei Medical University. All animal experiments followed the guidelines of the experimental animal management committee of Hebei Medical University. The rats were housed under standard conditions (temperature, $22-24{ }^{\circ} \mathrm{C}$, relative humidity, $45-55 \%$, and light, $12 \mathrm{~h}$ dark $12 \mathrm{~h}$ light cycle) for 5 days prior to use, and fasted $12 \mathrm{~h}$ before experiment, but free to water.

The experimental rats were randomly divided into 12 groups with 3 rats per group (group 1-2, the blank blood sample groups; group 3-4, the blank bile sample groups; group $5-6$, the blank urine and feces sample groups; group $7-8$, the experimental blood sample groups; group 9-10, the experimental bile sample groups; group 11-12, the experimental urine and feces sample groups). A certain amount of IGG was dissolved in $0.5 \%$ CMC-Na aqueous solution and mixed by ultrasonic to obtain IGG suspension. A certain amount of freeze-dried micelle powder was taken and dissolved in $0.5 \% \mathrm{CMC}-\mathrm{Na}$ aqueous solution, and the content of IGG was the same as that in IGG suspension. The blank micelle solution with the same concentration was prepared by the same method. The gavage volume was $16 \mathrm{~mL} / \mathrm{kg}$. The prepared IGG suspension was orally administered to 9 rats of group 7,9 and 11 at a dose of $100 \mathrm{mg} / \mathrm{kg}$, and the prepared IGG-loaded TPGS/ soluplus mixed micelles was orally administered to 9 rats of group 8,10 and 12 at a dose of $100 \mathrm{mg} / \mathrm{kg}$ (equal to the IGG). Group 1, 3 and 5 were given aqueous solution of $0.5 \% \mathrm{CMC}-\mathrm{Na}$, and group 2,4 and 6 were given blank micelle of the same concentration as the IGG-loaded micelle.

2.2.10.3. Bio-sample collection. Plasma samples: Approximately 300-500 $\mu \mathrm{L}$ blood was collected from the canthus of each rat at $5 \mathrm{~min}, 10 \mathrm{~min}$, $15 \mathrm{~min}, 30 \mathrm{~min}, 1 \mathrm{~h}, 2 \mathrm{~h}, 3 \mathrm{~h}, 6 \mathrm{~h}, 9 \mathrm{~h}, 12 \mathrm{~h}$ and $24 \mathrm{~h}$ after gavage. The blood samples were centrifuged at $1920 \times g$ for $10 \mathrm{~min}$ to obtain the plasma, and then the samples were merged.

Bile samples: After intragastric administration, each rat was subcutaneously injected with urethane solution at a dose of $1.0 \mathrm{~g} / \mathrm{kg}$. After anesthesia, the bile duct was intubated and the bile was collected for $24 \mathrm{~h}$.

Feces and urine samples: The rats were put into metabolic cages cage after administration, fasted, but can drink freely. The urine and feces samples were collected at regular intervals for $72 \mathrm{~h}$, and the urine and feces were merged, respectively [26].

2.2.10.4. Bio-sample pretreatment. For plasma, bile and urine pretreatment, an aliquot of $2 \mathrm{~mL}$ biosample was vortexed with $6 \mathrm{~mL}$ of methanol for $5 \mathrm{~min}$ and centrifuged for $10 \mathrm{~min}$ at $4{ }^{\circ} \mathrm{C}$ and 21,380 $\times g$. The supernatant was taken and dried under nitrogen gas at room temperature.

For feces, $6 \mathrm{~mL}$ methanol was added to $1 \mathrm{~g}$ feces, ultrasonically extracted for $30 \mathrm{~min}$ at $25{ }^{\circ} \mathrm{C}$ after vortexed, centrifuged at 21,380 $\times g$ for $10 \mathrm{~min}$, and the supernatant was taken. Repeat the above process once, and the supernatant was merged. The supernatants were evaporated to dryness under nitrogen gas. 
Before analysis, the residue was dissolved with $100 \mathrm{~mL}$ methanol, vortexed and centrifuged twice at $21,380 \times g$ for $10 \mathrm{~min}$. The supernatant was injected for analysis [26].

\section{Results}

\subsection{Determination of $\mathrm{CMC}$}

CMC is an important index to evaluate the stability of micelles. In this experiment, the CMC of mixed nanomicelles was determined by iodine hydrophobic probe method. The results showed that when the mass ratios of TPGS and soluplus were $0: 4,1: 3,2: 2$ and 3:1, the CMC of the mixed nanomicelles were all low, and when TPGS/soluplus was 1:3, the CMC of the mixed nanomicelles was the lowest, indicating that the mixed nanomicelles prepared by this ratio had the best stability. In addition to its high stability, because of its low $\mathrm{CMC}$, it can maintain its integrity even when diluted in the blood circulation was relatively insensitive to dilution, and has a longer circulation time compared with the surfactant micelles in vivo.

\subsection{Optimization of preparation conditions}

The CMC of the mixed nanomicelles was the lowest when TPGS/soluplus was 1:3, so the mass ratio of IGG, TPGS and soluplus was optimized on the basis of TPGS/soluplus was 1:3, and the results were shown in Table 1 . It could be seen from the table that when the ratio of IGG, TPGS and soluplus was 1:10:30, the encapsulation efficiency of micelles was the highest, the particle size was smaller, and the PDI was the smallest. Therefore, the ratio of 1:10:30 was chosen to prepare micelles.

\subsection{Determination of particle size, Zeta potential, encapsulation efficiency and drug loading}

Three batches of IGG-loaded TPGS/soluplus mixed micelles were prepared according to the ratio of IGG:TPGS:soluplus =1:10:30. The particle size of the micelles was $62.34 \pm 1.10 \mathrm{~nm}$ with a polydispersity index of $0.054 \pm 0.003$. The average Zeta potential measured was $-0.35 \pm 0.18 \mathrm{mV}$. The encapsulation efficiency and drug loading were $96.92 \pm 0.66 \%$ and $2.42 \pm 0.02 \%$, respectively. It has reported that neutral or slightly negative charged nanomedicines, which have much lower opsonization rate, present prolonged circulation in the bloodstream [21]. Therefore, the mixed nanomicelles can increase the circulation time of IGG in the blood and extend the time for it to play its pharmacological effects.

\subsection{Micelle stability}

The prepared IGG-loaded mixed micelles were stored at $4{ }^{\circ} \mathrm{C}$ for 60 days. The particle size, Zeta potential and encapsulation efficiency of micelles were measured at $0,15,30$ and 60 days, respectively. The clarity of micelle solution was observed. As presented in Table 2, the particle size and PDI did not change significantly, indicating there was no polymerization between micelle particles. From the encapsulation efficiency, it can be seen that the structure of the micelle was stable, it was difficult to self-degrade, and the drug did not break down and precipitate. In addition, the Zeta potential has not changed significantly. When visually examined, the micelle solution was still transparent and clear, and no precipitation was formed. The above results showed that the IGG-loaded mixed micelles could be kept relatively stable at $4{ }^{\circ} \mathrm{C}$ for a certain period of time.

\subsection{In vitro cytotoxicity}

In vitro cytotoxicity was carried out on A549 cells. The fatality rate of A549 cells incubated with different concentrations of IGG and IGG-loaded mixed micelles for $24 \mathrm{~h}$ were measured. IGG formulated in mixed micelles had better effects against the cancer cells than free drug after $24 \mathrm{~h}$ of incubation. The $\mathrm{IC}_{50}$ values of IGG and IGG-loaded mixed micelles calculated by GraphPad Prism 5 were $11.26 \pm 0.78 \mu \mathrm{g} / \mathrm{mL}$ and $3.47 \pm 0.01 \mu \mathrm{g} / \mathrm{mL}$, respectively, indicating that the cytotoxicity of the micelles to A549 cells was significantly enhanced.

Table 2. Characteristics of IGG-loaded TPGS/Soluplus mixed nanomicelles stored for $0 d, 15 d, 30 d$ and $60 d$.

\begin{tabular}{lllll}
\hline & $0 \mathrm{~d}$ & $15 \mathrm{~d}$ & $30 \mathrm{~d}$ & $60 \mathrm{~d}$ \\
\hline Size $(\mathrm{nm})$ & $62.34 \pm 1.10$ & $63.57 \pm 1.13$ & $62.25 \pm 1.47$ & $64.62 \pm 0.48$ \\
PDI & $0.054 \pm 0.003$ & $0.050 \pm 0.026$ & $0.057 \pm 0.027$ & $0.044 \pm 0.010$ \\
Zeta Potential $(\mathrm{mV})$ & $-0.35 \pm 0.18$ & $-0.55 \pm 0.19$ & $-1.39 \pm 1.62$ & $0.64 \pm 0.17$ \\
EE $(\%)$ & $96.92 \pm 0.66$ & $96.13 \pm 0.17$ & $96.17 \pm 0.24$ & $96.12 \pm 0.46$ \\
Clarity & clear & clear & clear & clear \\
\hline
\end{tabular}




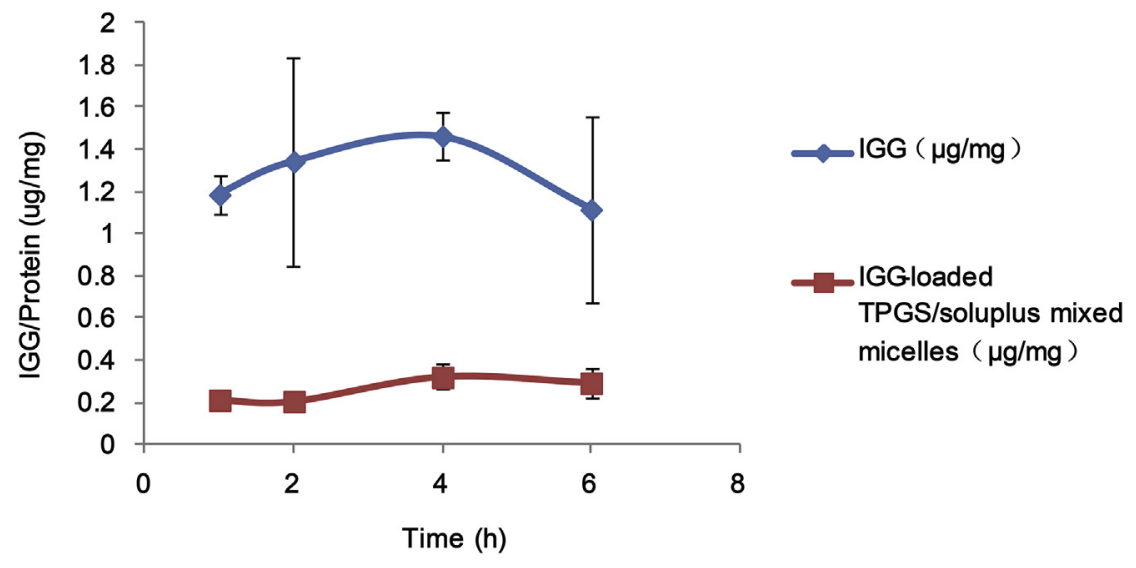

Fig. 1. Time-dependent intracellular uptake in A549 cell lines for IGG and IGG-loaded TPGS/soluplus mixed nanomicelles. Drug amount was normalized by protein concentrations of the cell lysates. Results are expressed as mean \pm S.D. $(n=3)$.

$1 \mathrm{~h}$

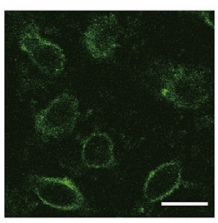

IGG
$2 \mathrm{~h}$
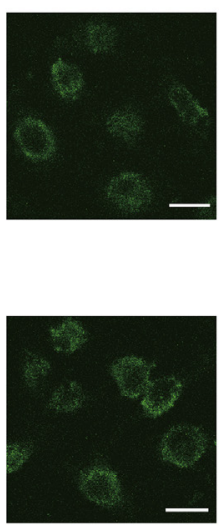

$4 \mathrm{~h}$
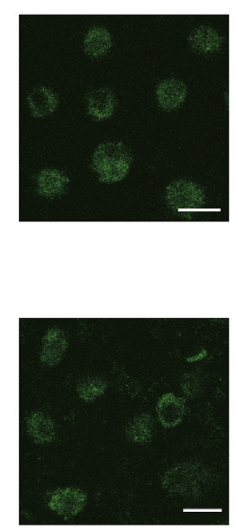

$6 \mathrm{~h}$
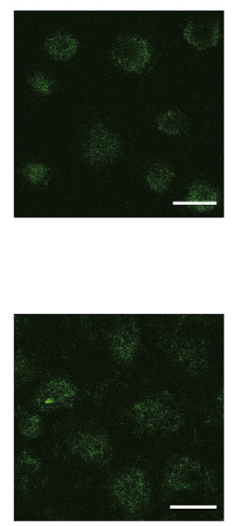

Fig. 2. Cellular uptake of IGG and IGG-loaded TPGS/soluplus mixed micelles in A549 cells observed by laser confocal microscope. Scale bar is $20 \mu$ m.

\subsection{In vitro cellular uptake}

The cellular uptake plays significant roles in the antitumor effect of drug delivery. To examine whether IGG-loaded TPGS/Soluplus mixed micelles can be internalized, the cellular uptake of mixed nanomicelles of IGG and IGG-loaded mixed nanomicelles was studied quantitatively and qualitatively by HPLC and laser confocal microscopy. The quantitative results were shown in the Fig. 1. It can be seen from the figure that the uptake of IGG and IGG-loaded mixed micelles increased with the increase of time within 1-4 $\mathrm{h}$, and decreased significantly after $4 \mathrm{~h}$, and the intake of monomers was significantly higher than that of micelles $(p<0.01)$, which may be related to the negative charge of micelles and cell membrane surface. It can be seen from the confocal picture in Fig. 2 that the uptake of IGG and IGG-loaded mixed micelles increased significantly in $1-4 \mathrm{~h}$, but after $4 \mathrm{~h}$, due to the toxic effect of the drug on the cells, the cell morphology began to change, the cells began to crack gradually, and the drug began to release, so the drug content in the cells began to decrease, which was similar to the quantitative results preceding.

\subsection{Analysis of the metabolites of IGG and IGG- loaded TPGS/soluplus mixed micelles}

\subsubsection{Metabolites of IGG}

In this study, 32 metabolites of IGG were detected in rats. The result was shown in Fig. 3 and Table 3. They were mainly distributed in feces. No metabolites of IGG was detected in plasma, bile and urine, which indicated that the bioavailability of IGG was very low, and it was difficult for IGG to enter the blood circulation to play a pharmacological role. Among the metabolites in rats, 16 were phase Imetabolites and 16 were phase II metabolites. The main 


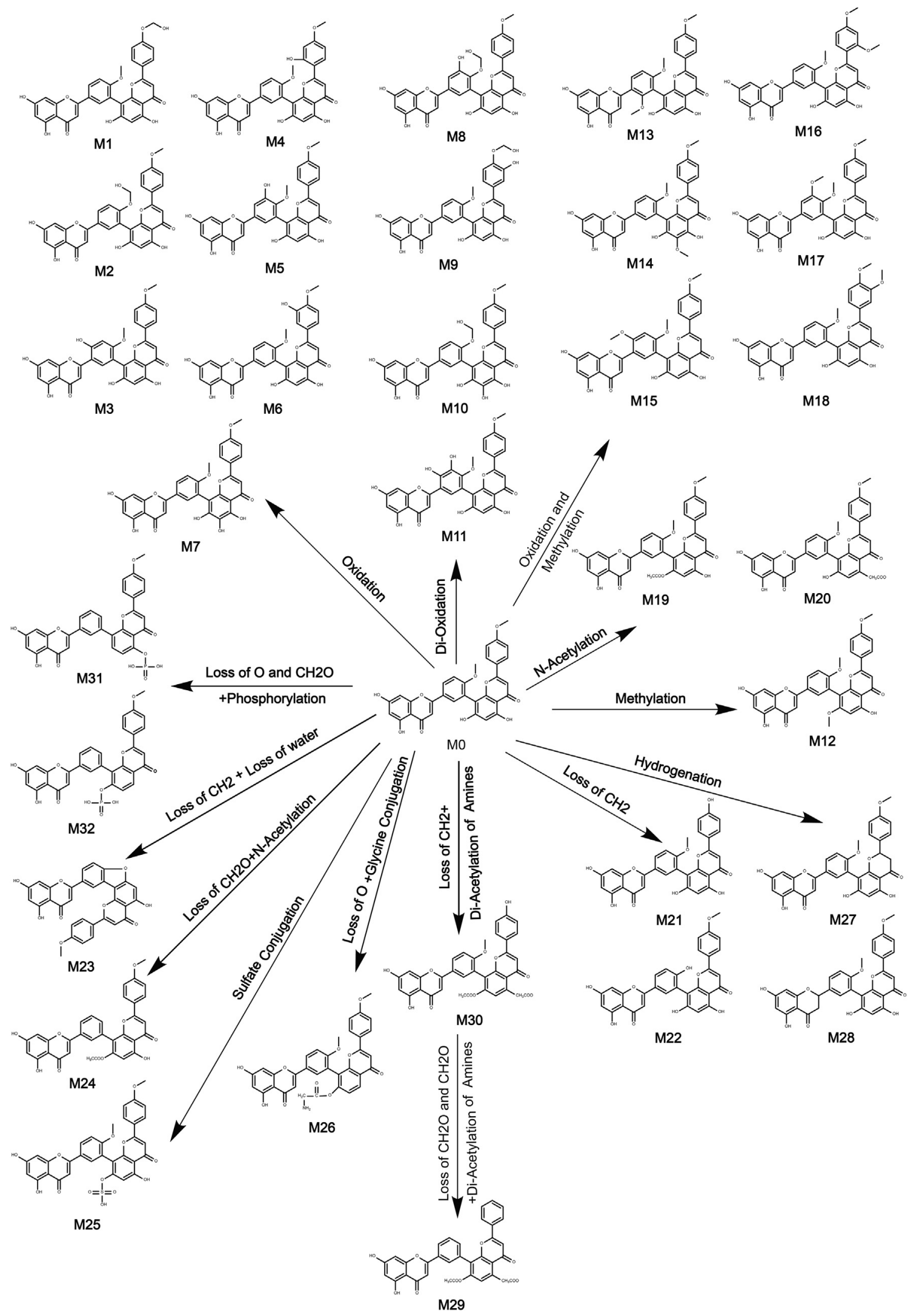

Fig. 3. Metabolic profile and proposed metabolic pathways of IGG in rats. 
Table 3. Summary of metabolites of IGG and IGG-loaded TPGS/soluplus mixed nanomicelles in rats.

\begin{tabular}{|c|c|c|c|c|c|c|c|c|c|c|c|}
\hline $\begin{array}{l}\text { Metabolite } \\
\text { ID }\end{array}$ & Name & Formula & $m / z$ & ppm & R.T.(min) & MS/MS Fragments & Clog P & Plasma & Bile & Urine & $\mathrm{Fec}$ \\
\hline M1 & Oxidation & $\mathrm{C}_{32} \mathrm{H}_{22} \mathrm{O}_{11}$ & 581.1085 & -2.5 & 10.05 & $513.1199,415.0484,191.0933,133.0273$ & 4.8329 & - & - & - & + \\
\hline M2 & Oxidation & $\mathrm{C}_{32} \mathrm{H}_{22} \mathrm{O}_{11}$ & 581.1095 & 1 & 10.23 & $535.0677,513.2951,389.2664,187.1029$ & 4.8329 & - & - & - & + \\
\hline M3 & Oxidation & $\mathrm{C}_{32} \mathrm{H}_{22} \mathrm{O}_{11}$ & 581.1062 & -4.6 & 10.63 & $553.1212,537.1285,513.1214,425.0632$ & 5.0768 & - & - & - & + \\
\hline M4 & Oxidation & $\mathrm{C}_{32} \mathrm{H}_{22} \mathrm{O}_{11}$ & 581.1081 & -1.4 & 11.12 & $535.3074,513.2664,389.2800$ & 5.0883 & - & - & - & + \\
\hline M5 & Oxidation & $\mathrm{C}_{32} \mathrm{H}_{22} \mathrm{O}_{11}$ & 581.1094 & 0.7 & 11.32 & $533.0922,505.0928,389.0670,151.0050$ & 5.1268 & - & - & - & + \\
\hline M6 & Oxidation & $\mathrm{C}_{32} \mathrm{H}_{22} \mathrm{O}_{11}$ & 581.1095 & 0.7 & 11.69 & $537.1227,513.1194,425.0716,175.0382$ & 5.1383 & - & - & - & + \\
\hline M7 & Oxidation & $\mathrm{C}_{32} \mathrm{H}_{22} \mathrm{O}_{11}$ & 581.1094 & 0.5 & 11.87 & $549.0857,513.1215,389.0675,123.0083$ & 5.3117 & - & - & - & + \\
\hline M8 & Di-Oxidation & $\mathrm{C}_{32} \mathrm{H}_{22} \mathrm{O}_{12}$ & 597.1025 & -2.3 & 8.69 & $579.0974,569.1139,465.0978,123.0449$ & 3.7348 & - & - & - & + \\
\hline M9 & Di-Oxidation & $\mathrm{C}_{32} \mathrm{H}_{22} \mathrm{O}_{12}$ & 597.1036 & -0.5 & 9.2 & $579.2418,569.1080,529.3027,147.0272$ & 4.0848 & - & - & - & + \\
\hline M10 & Di-Oxidation & $\mathrm{C}_{32} \mathrm{H}_{22} \mathrm{O}_{12}$ & 597.1047 & 1.4 & 10.07 & $579.0946,529.2619,389.1045,357.0721$ & 4.0963 & - & - & - & + \\
\hline M11 & Di-Oxidation & $\mathrm{C}_{32} \mathrm{H}_{22} \mathrm{O}_{12}$ & 597.1037 & -0.3 & 11.69 & $465.1006,139.0029,107.0113$ & 4.1605 & - & - & - & + \\
\hline M12 & Methylation & $\mathrm{C}_{33} \mathrm{H}_{24} \mathrm{O}_{10}$ & 579.1290 & -1.2 & 14.61 & $533.3777,511.2749,503.0797,429.0624$ & 5.561 & - & - & - & + \\
\hline M13 & Oxidation and Methylation & $\mathrm{C}_{33} \mathrm{H}_{24} \mathrm{O}_{11}$ & 595.1246 & 0.1 & 10.28 & $563.1012,533.0907,527.2809,151.0398$ & 4.8317 & - & - & - & + \\
\hline M14 & Oxidation and Methylation & $\mathrm{C}_{33} \mathrm{H}_{24} \mathrm{O}_{11}$ & 595.1265 & 3.3 & 11.22 & $563.1021,533.0907,527.3069,151.0036$ & 5.38 & - & - & - & + \\
\hline M15 & Oxidation and Methylation & $\mathrm{C}_{33} \mathrm{H}_{24} \mathrm{O}_{11}$ & 595.1251 & 0.9 & 11.52 & $547.0708,533.0929,527.3057,107.0158$ & 5.3917 & - & - & - & + \\
\hline M16 & Oxidation and Methylation & $\mathrm{C}_{33} \mathrm{H}_{24} \mathrm{O}_{11}$ & 595.1242 & -0.7 & 11.86 & $547.0687,489.3638,527.2543,151.0027$ & 5.4015 & - & - & - & + \\
\hline M17, & Oxidation and Methylation & $\mathrm{C}_{33} \mathrm{H}_{24} \mathrm{O}_{11}$ & 595.1265 & 3.2 & 12.12 & $533.2872,511.3083,415.0429,151.1162$ & 5.6017 & - & - & - & + \\
\hline M18 & Oxidation and Methylation & $\mathrm{C}_{33} \mathrm{H}_{24} \mathrm{O}_{11}$ & 595.1256 & 1.7 & 12.71 & $489.0638,527.1367,389.0290,151.0053$ & 5.6115 & - & - & - & + \\
\hline M19 & N-Acetylation & $\mathrm{C}_{34} \mathrm{H}_{24} \mathrm{O}_{11}$ & 607.1237 & -1.5 & 13.87 & $565.1166,563.3634,539.3171,389.0574$ & 5.6226 & - & - & - & + \\
\hline M20 & $\mathrm{N}$-Acetylation & $\mathrm{C}_{34} \mathrm{H}_{24} \mathrm{O}_{11}$ & 607.1245 & -0.1 & 14.28 & $575.0980,539.3226,533.0984,425.2736$ & 5.891 & - & - & - & + \\
\hline M21 & Loss of $\mathrm{CH}_{2}$ & $\mathrm{C}_{31} \mathrm{H}_{20} \mathrm{O}_{10}$ & 551.0980 & -0.6 & 12.08 & $465.1010,433.0986,389.0664,151.0375$ & 5.2939 & - & - & - & + \\
\hline M22 & Loss of $\mathrm{CH}_{2}$ & $\mathrm{C}_{31} \mathrm{H}_{20} \mathrm{O}_{10}$ & 551.0990 & 1.1 & 12.36 & $533.3093,519.0712,505.3546,483.1102$ & 5.5375 & - & - & - & + \\
\hline M23 & Loss of $\mathrm{CH}_{2}+$ Loss of water & $\mathrm{C}_{31} \mathrm{H}_{18} \mathrm{O}_{9}$ & 533.0879 & 0.1 & 11.69 & $517.0587,489.0982,401.0317,381.0784$ & 7.4093 & - & - & - & + \\
\hline M24 & Loss of $\mathrm{CH}_{2} \mathrm{O}+\mathrm{N}$-Acetylation & $\mathrm{C}_{33} \mathrm{H}_{22} \mathrm{O}_{10}$ & 577.1132 & -1.5 & 8.50 & $547.1056,545.0899,509.2596,325.2170$ & 6.2511 & - & - & - & + \\
\hline M25 & Sulfate Conjugation & $\mathrm{C}_{32} \mathrm{H}_{22} \mathrm{O}_{13} \mathrm{~S}$ & 645.0719 & 1.7 & 9.48 & $587.3232,577.2863,533.0906,389.0673$ & 4.6066 & - & - & - & + \\
\hline M26 & Loss of $\mathrm{O}+$ Glycine Conjugation & $\mathrm{C}_{34} \mathrm{H}_{25} \mathrm{NO}_{10}$ & 606.1414 & 2.0 & 11.69 & $547.1088,538.1405,518.0672,480.1015$ & 4.0581 & - & - & - & + \\
\hline M27 & Hydrogenation & $\mathrm{C}_{32} \mathrm{H}_{24} \mathrm{O}_{10}$ & 567.1302 & 1.0 & 12.04 & $499.1434,491.1174,451.1205,307.0627$ & 5.408 & - & - & - & + \\
\hline M28 & Hydrogenation & $\mathrm{C}_{32} \mathrm{H}_{24} \mathrm{O}_{10}$ & 567.1308 & 1.9 & 13.06 & $535.0823,499.3127,415.0910,175.0375$ & 5.4159 & - & - & - & + \\
\hline M29 & $\begin{array}{l}\text { Loss of } \mathrm{CH} 2 \mathrm{O} \text { and } \\
\mathrm{CH} 2 \mathrm{O}+\mathrm{Di}-\text { Acetylation } \\
\text { of Amines }\end{array}$ & $\mathrm{C}_{34} \mathrm{H}_{22} \mathrm{O}_{10}$ & 589.1131 & -1.6 & 12.04 & $547.1046,515.0738,479.1144,261.1251$ & 6.2643 & - & - & - & + \\
\hline M30 & $\begin{array}{l}\text { Loss of } \mathrm{CH} 2+\text { Di-Acetylation } \\
\text { of Amines }\end{array}$ & $\mathrm{C}_{35} \mathrm{H}_{24} \mathrm{O}_{12}$ & 635.1214 & 2.9 & 12.63 & $603.1034,567.2791,499.3158,389.0701$ & 5.0045 & - & - & - & + \\
\hline M31 & $\begin{array}{l}\text { Loss of } \mathrm{O} \text { and } \\
\mathrm{CH}_{2} \mathrm{O}+\text { Phosphorylation }\end{array}$ & $\mathrm{C}_{31} \mathrm{H}_{21} \mathrm{O}_{11} \mathrm{P}$ & 599.0757 & 1.4 & 12.44 & $567.0526,531.0738,503.0798,357.0817$ & 4.3758 & - & - & - & + \\
\hline M32 & $\begin{array}{l}\text { Loss of } \mathrm{O} \text { and } \\
\mathrm{CH}_{2} \mathrm{O}+\text { Phosphorylation }\end{array}$ & $\mathrm{C}_{31} \mathrm{H}_{21} \mathrm{O}_{11} \mathrm{P}$ & 599.0781 & 5.4 & 13.15 & $531.2863,517.3174,483.2679,151.0041$ & 4.3758 & - & - & - & + \\
\hline N1 & Oxidation & $\mathrm{C}_{32} \mathrm{H}_{22} \mathrm{O}_{11}$ & 581.1093 & 0.6 & 10.23 & $549.0852,513.1198,415.0462,151.0030$ & 4.8329 & - & - & - & + \\
\hline $\mathrm{N} 2$, & Oxidation & $\mathrm{C}_{32} \mathrm{H}_{22} \mathrm{O}_{11}$ & 581.1100 & 1.8 & 11.12 & $537.1206,513.3045,429.0441,389.0634$ & 5.08829 & - & - & - & + \\
\hline N3 & Oxidation & $\mathrm{C}_{32} \mathrm{H}_{22} \mathrm{O}_{11}$ & 581.1089 & -0.1 & 11.67 & $565.1115,553.1118,513.1195,389.0644$ & 5.13829 & - & - & + & + \\
\hline $\mathrm{N} 4$ & Oxidation & $\mathrm{C}_{32} \mathrm{H}_{22} \mathrm{O}_{11}$ & 581.1082 & -1.2 & 11.81 & 563.0931, 497.3011,437.0159 & 5.31166 & - & + & - & - \\
\hline N5 & Di-Oxidation & $\mathrm{C}_{32} \mathrm{H}_{22} \mathrm{O}_{12}$ & 597.1048 & 1.7 & 11.69 & $579.0855,529.3285,463.2322,139.0049$ & 4.16048 & - & - & - & + \\
\hline N6 & Loss of $\mathrm{CH}_{2}$ & $\mathrm{C}_{31} \mathrm{H}_{20} \mathrm{O}_{10}$ & 551.0979 & -0.8 & 12.08 & $519.0724,507.1089,399.0535,389.0687$ & 5.29394 & - & - & - & + \\
\hline N7 & Loss of $\mathrm{CH}_{2}$ & $\mathrm{C}_{31} \mathrm{H}_{20} \mathrm{O}_{10}$ & 551.0956 & -5 & 12.41 & $535.0635,533.0444,483.1062,465.0316$ & 5.53754 & - & - & + & + \\
\hline
\end{tabular}




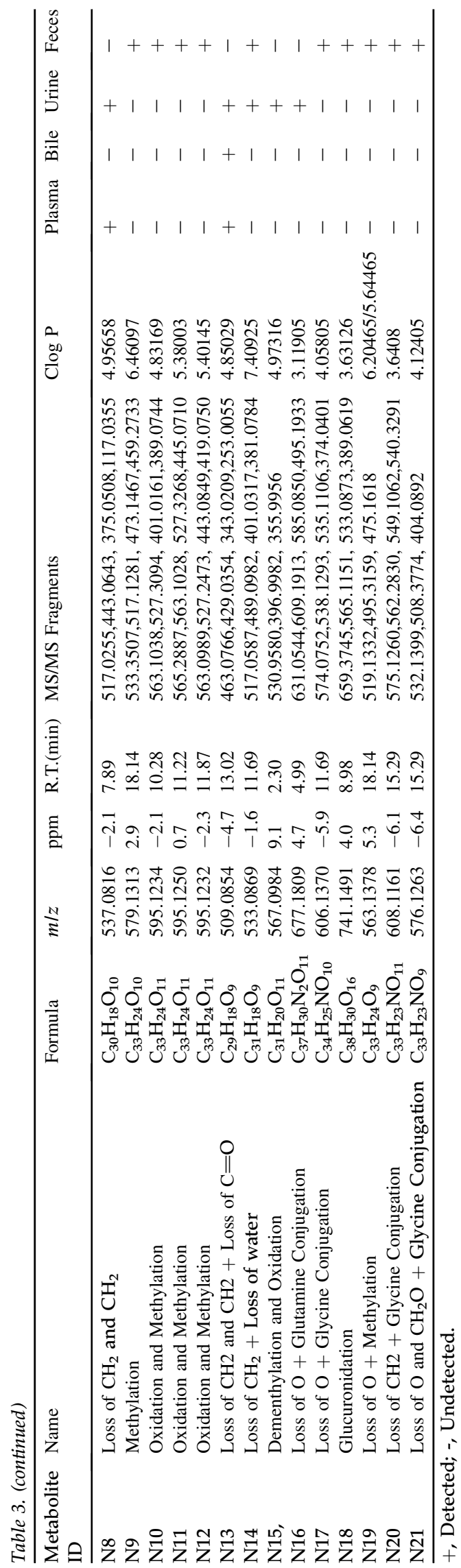

metabolic pathways were oxidation, di-oxidation, oxidation and methylation, acetylation and hydrogenation, among which oxidation, di-oxidation and oxidation and methylation were the main reaction pathways, which coincided with the strong antioxidant activity of IGG.

\subsubsection{Metabolites of IGG-loaded TPGS/soluplus mixed micelles}

In this experiment, 21 kinds of metabolites of IGG-loaded micelles were detected in rats, including 2 in plasma (N8, N13), 2 in bile (N4, N13), 7 in urine (N13, N7, N8, N13 N16) and 16 in faeces (N1 N3, N5 N7, N9 N12, N14, N17 N21). The results were shown in Fig. 4 and Table 3 . It can be seen from Table 3 that no metabolites in plasma and bile are detected in the feces, and only three of the same metabolites in urine were detected in the feces. This indicated that the difference in matrix led to different interactions between various substances, which led to different metabolic pathways, and the resulting metabolites were also different. Among these metabolites, there were 11 species of phase I metabolites and 10 species of phase II metabolites. The metabolic pathways were mainly oxidation, oxidation and methylation and demethylation.

\section{Discussion}

In this study, after condition optimization, IGGloaded TPGS/soluplus mixed nanomicelles were successfully prepared with a small particle size, uniform distribution, and high encapsulation efficiency, but the drug loading was low, which was caused by the large molecular mass of TPGS and soluplus. The IGG-loaded mixed nanomicelle showed good stability, which was determined by the structure of the micelle. The drugs was enclosed in the core region of the micelle by the carriers, so that the drugs could not be degraded by various biochemical substances in the matrix, such as gastric acid and P450 enzymes [27], which ensured that the drug could enter the blood circulation through the gastrointestinal wall in the form of micelles, and also delay the release of the drug to play a pharmacological role for a longer period of time.

And in this study we found that IGG-loaded mixed nanomicelles showed higher cytotoxicity than monomer, about 3.5 times that of monomer, which may be related to the toxicity of drug carrier TPGS to cancer cells. The anticancer activity of TPGS was reported to be associated to its apoptosis-inducing properties by the generation of reactive oxygen species, which might be the reason for the toxicity of high concentration of blank micelles to cancer cells [22]. It 

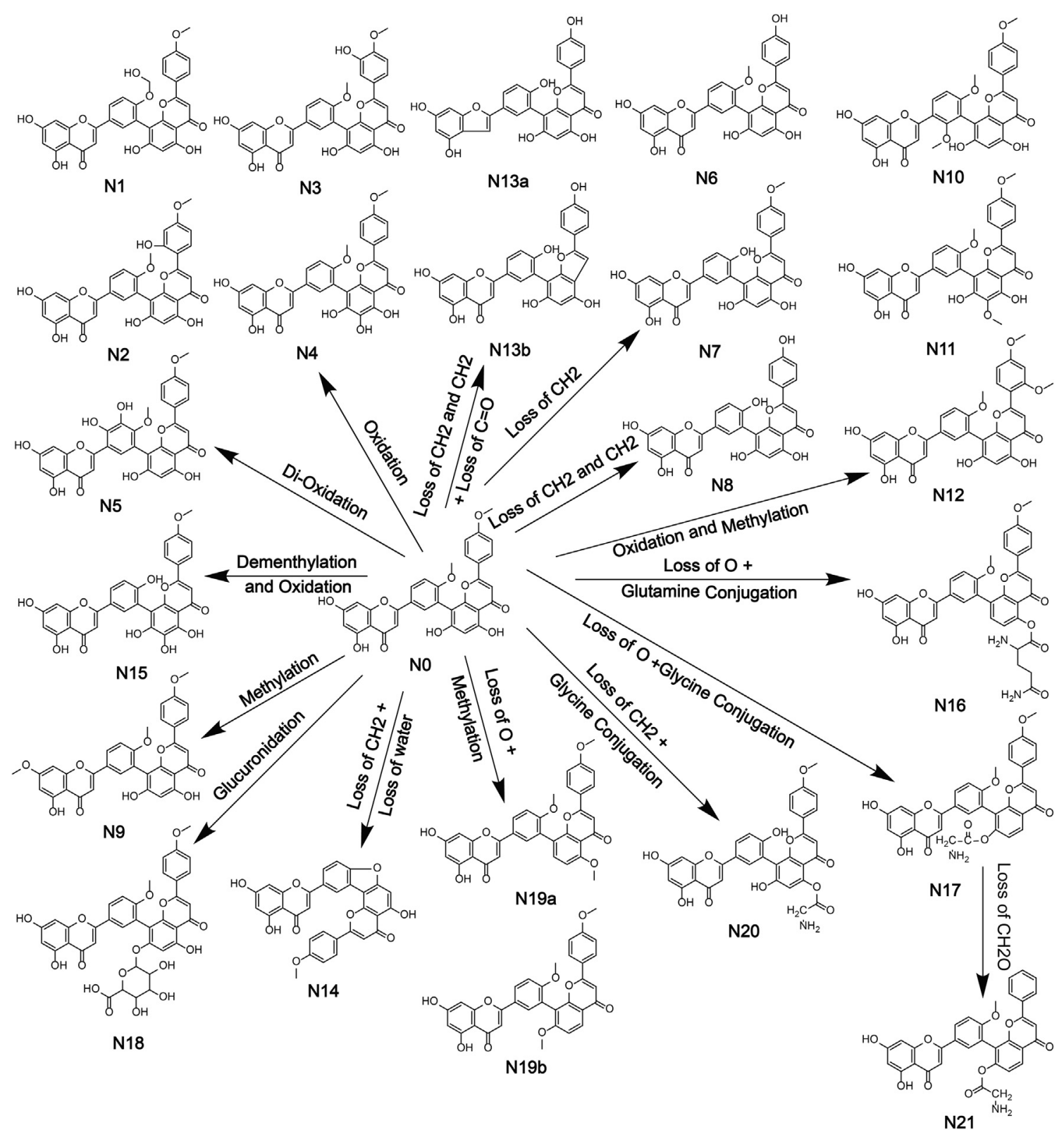

Fig. 4. Metabolic profile and proposed metabolic pathways of IGG-loaded TPGS/soluplus mixed nanomicelles in rats.

also indicated that TPGS played an important role in increasing drug toxicity, and the high cytotoxicity of the micelles may be related to the combined action of the carrier and the drugs [25]. According to our previous studies, the cytotoxicity of IGG was significantly stronger than that of amentoflavone, which was due to the fact that the methoxy group at the position of $C-4$ in ring $B$ exhibited stronger antiproliferative activity, and it was consistent with the better anti-inflammatory activity [9].

In the experiments of cytotoxicity and cellular uptake, we found that there was no positive correlation between cytotoxicity and cellular uptake. The increased cytotoxicity may be caused by TPGS rather than the increase of cellular uptake; or maybe IGG can regulate some receptors on the cell surface and lead to cell apoptosis. All these need further study.

Among the 32 metabolites of IGG, there were 17 kinds of oxidation, di-oxidation and oxidation and methylation metabolites, indicating that IGG have strong antioxidant activity, which was consistent with previous reports [22]. Among the metabolites of IGG-loaded mixed nanomicelles, the metabolites in plasma, bile, urine and feces were also different, indicating that in different substrates, the metabolites were different. 
In the metabolism study of nanomicelles and monomers, the metabolites of nanomicelles were significantly less than those of monomers, which was due to the stable structure of micelles. When the drugs were encapsulated, it was not easily degraded by proteases and other biochemical substances in the gastrointestinal tract. Thereby, the metabolites in feces were reduced. In addition, from the distribution of metabolites, we can detect the metabolites of IGGloaded mixed nanomicelles in feces, urine, bile and plasma, but for monomers, we can only detect metabolites in feces, which meant that IGG was hardly dissolved in water and could not be effectively absorbed and utilized in the gastrointestinal tract, and its bioavailability was very low.

But, when it was prepared into micelles, IGG could be absorbed into blood, bile and urine, and the metabolites were enough to be detected. By comparing the metabolites of IGG monomers and mixed nanomicelles, it was found that among the metabolites of micelles, there were 3 metabolites in plasma bile and urine different from that of the monomers, which was due to the difference in the matrix. And five kinds of metabolites in feces were different from that of monomers. It indicated that the metabolic pathway of monomers in feces had also changed after being prepared into micelles, resulting in the difference of metabolites.

In this experiment, we prepared an IGG mixed nanomicelle with good solubility and stability, high encapsulation efficiency, and a particle size of less than $100 \mathrm{~nm}$, which was highly toxic to cancer cells and released slowly in the body. It can provide a good choice for the development of anticancer drugs.

\section{Conclusion}

In this experiment, IGG-loaded TPGS/soluplus mixed micelles with small particle size, uniform distribution and high encapsulation efficiency were successfully prepared. The physicochemical properties could be relatively stable within $60 \mathrm{~d}$, and the toxicity to A549 cells was significantly higher than that of IGG monomer. However, the cellular uptake was lower than that of IGG monomers due to the negative electricity on the surface of micelles and cell membranes. In the metabolism, a total of 32 metabolites of IGG monomer and 21 metabolites of IGG-loaded mixed micelles were detected. The metabolites of IGG can only be detected in feces while the metabolites of IGG-loaded mixed micelles can also be detected in feces, urine, bile and plasma, which indicated that he bioavailability of IGG was improved after being prepared into mixed nanomicelles and the metabolite pathway was changed.
The results were shown in Table 3 . In conclusion, after the preparation of nano-micelles, the solubility and stability were enhanced, cytotoxicity was increased and the bioavailability was improved, which provided a new choice for the development of new drugs.

\section{Conflict of interest}

All the authors have declared no conflict of interest.

\section{Acknowledgement}

The project was financially supported by the $\mathrm{Na}$ tional Natural Science Foundation of China (No. 81473180), Youth Foundation of Hebei Educational Committee (No. QN2018069) and the Natural Science Foundation of Hebei Province, China (No. H2019206189). Thanks to the Department of Pharmaceutical Analysis, School of Pharmacy, Hebei Medical University for the instrument support.

\section{Appendix A. Supplementary data}

Supplementary data to this article can be found online at https://doi.org/10.38212/2224-6614.1065.

\section{References}

[1] Peer D, Karp JM, Hong S, FaroKHzad OC, Margalit R, Langer R. Nanocarriers as an emerging platform for cancer therapy. Nat Nanotechnol 2007;2:751-60.

[2] Mekhail TM, Markman M. Paclitaxel in cancer therapy. Expet Opin Pharmacother 2002;3:755-66.

[3] Nakhjavani M, Hardingham JE, Palethorpe HM, Tomita Y, Smith E, Price TJ, et al. Ginsenoside Rg3: potential molecular targets and therapeutic indication in metastatic breast cancer. Medicines (Basel) 2019;6.

[4] Vervandier-Fasseur D, Latruffe N. The potential use of resveratrol for cancer prevention. Molecules 2019;24.

[5] Li X, Ouyang X, Cai R, Chen D. 3',8"-Dimerization enhances the antioxidant capacity of flavonoids: evidence from acacetin and isoginkgetin. Molecules 2019;24.

[6] Bagla VP, McGaw LJ, Elgorashi EE, Eloff JN. Antimicrobial activity, toxicity and selectivity index of two biflavonoids and a flavone isolated from Podocarpus henkelii (Podocarpaceae) leaves. BMC Compl Alternative Med 2014;14.

[7] Vanzyl EJ, Rick KRC, Blackmore AB, MacFarlane EM, McKay BC. Flow cytometric analysis identifies changes in S and $\mathrm{M}$ phases as novel cell cycle alterations induced by the splicing inhibitor isoginkgetin. PloS One 2018;13:e0191178.

[8] Yoon SO, Shin S, Lee HJ, Chun HK, Chung AS. Isoginkgetin inhibits tumor cell invasion by regulating phosphatidylinositol 3-kinase/Akt-dependent matrix metalloproteinase-9 expression. Mol Canc Therapeut 2006;5:2666-75.

[9] Li M, Li B, Xia ZM, Tian Y, Zhang D, Rui WJ, et al. Anticancer effects of five biflavonoids from Ginkgo biloba L. Male flowers in vitro. Molecules 2019;24.

[10] O'Brien K, Matlin AJ, Lowell AM, Moore MJ. The biflavonoid isoginkgetin is a general inhibitor of Pre-mRNA splicing. J Biol Chem 2008;283:33147-54.

[11] Tsalikis J, Abdel-Nour M, Farahvash A, Sorbara MT, Poon S, Philpott DJ, et al. Isoginkgetin, a natural biflavonoid 
proteasome inhibitor, sensitizes cancer cells to apoptosis via disruption of lysosomal homeostasis and impaired protein clearance. Mol Cell Biol 2019;39.

[12] Du X, Yin S, Xu L, Ma J, Yu H, Wang G, et al. Polylysine and cysteine functionalized chitosan nanoparticle as an efficient platform for oral delivery of paclitaxel. Carbohydr Polym 2020;229:115484.

[13] Zhang J, Zhou J, Zhang T, Niu Z, Wang J, Guo J, et al. Facile fabrication of an amentoflavone-loaded micelle system for oral delivery to improve bioavailability and hypoglycemic effects in KKAy mice. ACS Appl Mater Interfaces 2019;11: 12904-13.

[14] Katekar R, Thombre G, Riyazuddin M, Husain A, Rani H, Praveena KS, et al. Pharmacokinetics and Brain targeting of Trans-Resveratrol loaded mixed micelles in rats following intravenous administration. Pharmaceutical Development and Technology; 2019. p. 1-28.

[15] Shuai Q, Zhao G, Lian X, Wan J, Cen B, Zhang W, et al. Selfassembling poly(ethylene glycol)-block-polylactide-cabazitaxel conjugate nanoparticles for anticancer therapy with high efficacy and low in vivo toxicity. Int J Pharm 2019;574: 118879.

[16] Jain RK, Stylianopoulos T. Delivering nanomedicine to solid tumors. Nat Rev Clin Oncol 2010;7:653-64.

[17] Wu L, Zhao L, Su X, Zhang P, Ling G. Repaglinide-loaded nanostructured lipid carriers with different particle sizes for improving oral absorption: preparation, characterization, pharmacokinetics, and in situ intestinal perfusion. Drug Deliv 2019:1-10.

[18] Cagel M, Tesan FC, Bernabeu E, Salgueiro MJ, Zubillaga MB, Moretton MA, et al. Polymeric mixed micelles as nanomedicines: achievements and perspectives. Eur J Pharm Biopharm 2017;113:211-28.
[19] Kwon GS. Polymeric micelles for delivery of poorly watersoluble compounds. Crit Rev Ther Drug Carrier Syst 2003;20: 357-403.

[20] Gaucher G, Dufresne MH, Sant VP, Kang N, Maysinger D, Leroux JC. Block copolymer micelles: preparation, characterization and application in drug delivery. J Contr Release 2005;109:169-88.

[21] Cabral H, Miyata K, Osada K, Kataoka K. Block copolymer micelles in nanomedicine applications. Chem Rev 2018;118: 6844-92.

[22] Bernabeu E, Gonzalez L, Cagel M, Gergic EP, Moretton MA, Chiappetta DA. Novel Soluplus((R))-TPGS mixed micelles for encapsulation of paclitaxel with enhanced in vitro cytotoxicity on breast and ovarian cancer cell lines. Colloids Surf B Biointerfaces 2016;140:403-11.

[23] Jiang S, Mou Y, He H, Yang D, Qin L, Zhang F, et al. Preparation and evaluation of self-assembly Soluplus((R))-sodium cholate-phospholipid ternary mixed micelles of docetaxel. Drug Dev Ind Pharm 2019:1-11.

[24] Chen Y, Jiao Y, Ge YX, Liu GX, Xu W, Li LB. The study of tacrolimus-loaded polyion complex micelles for oral delivery. J Biomed Nanotechnol 2017;13:1147-57.

[25] Ding Y, Wang C, Wang Y, Xu Y, Zhao J, Gao M, et al. Development and evaluation of a novel drug delivery: soluplus((R))/TPGS mixed micelles loaded with piperine in vitro and in vivo. Drug Dev Ind Pharm 2018;44:1409-16.

[26] Chen Y, Feng X, Li L, Zhang X, Song K, Diao X, et al. UHPLC-Q-TOF-MS/MS method based on four-step strategy for metabolites of hinokiflavone in vivo and in vitro. J Pharmaceut Biomed Anal 2019;169:19-29.

[27] Chiappetta DA, Sosnik A. Poly(ethylene oxide)-poly(propylene oxide) block copolymer micelles as drug delivery agents: improved hydrosolubility, stability and bioavailability of drugs. Eur J Pharm Biopharm 2007;66:303-17. 